\title{
Multiscale modeling and topology optimization of poroelastic actuators
}

\section{Andreasen, Casper Schousboe; Sigmund, Ole}

\section{Published in:}

Smart Materials and Structures

Link to article, DOI:

10.1088/0964-1726/21/6/065005

Publication date:

2012

Link back to DTU Orbit

Citation (APA):

Andreasen, C. S., \& Sigmund, O. (2012). Multiscale modeling and topology optimization of poroelastic actuators. Smart Materials and Structures, 21(6), Paper 065005. https://doi.org/10.1088/0964-1726/21/6/065005

\section{General rights}

Copyright and moral rights for the publications made accessible in the public portal are retained by the authors and/or other copyright owners and it is a condition of accessing publications that users recognise and abide by the legal requirements associated with these rights.

- Users may download and print one copy of any publication from the public portal for the purpose of private study or research.

- You may not further distribute the material or use it for any profit-making activity or commercial gain

- You may freely distribute the URL identifying the publication in the public portal

If you believe that this document breaches copyright please contact us providing details, and we will remove access to the work immediately and investigate your claim. 


\title{
Multiscale modeling and topology optimization of poroelastic actuators
}

\author{
C.S. Andreasen, O. Sigmund \\ Department for Mechanical Engineering, Solid Mechanics, Technical University of \\ Denmark, Building 404, 2800 Kgs. Lyngby, Denmark \\ E-mail: csan@mek.dtu.dk
}

\begin{abstract}
This paper presents a method for design of optimized poroelastic materials which under internal pressurization turn into actuators for application in e.g. linear motors. The actuators are modeled in a two scale fluid-structure interaction approach. The fluid saturated material microstructure is optimized using topology optimization in order to achieve a better macroscopic performance quantified by vertical or torsional deflections. Constraints are introduced to ensure a certain deflection / extension ratio of the actuator.
\end{abstract}

\section{Introduction}

Poroelasticity, mostly applied in the field of earth mechanics, may also be used in the modeling and design of smart saturated porous actuators. This could for instance be a pressure driven linear motor in which the actuators are composed of sealed saturated poroelastic materials. Ordinary random microstructured poroelastic materials such as foams usually swell equally in all directions when pressurized. By designing the microstructure it is possible to make the structure deflect in a prescribed manner like e.g. bending or twisting. This could for example be of use in linear motors. Usually linear motors for use in e.g. atomic force microscopes use piezoelectric actuation cf. Friend et al. (2004), however, in a non-electronics environment the use of poroelastic actuators driven by a pressure change may be a viable alternative.

An illustration of the working principle of a linear motor is shown in figure 1 . The linear motor is based on a bi-morph actuator and works in a sequence of four steps. At first the actuator is at rest. Then one of the subdomains is pressurized and elongates which makes the actuator bend and grip the rod. The other subdomain starts to elongate due to pressurization while the first retracts as it is depressurized. This makes the actuator, and hence the actuation rod, move sideways. Finally, the second subdomain is depressurized and the rod has been translated an amount corresponding to the horizontal actuation distance of the actuator. The sequence is repeated and may with two actuators result in a linear motion with constant velocity. 


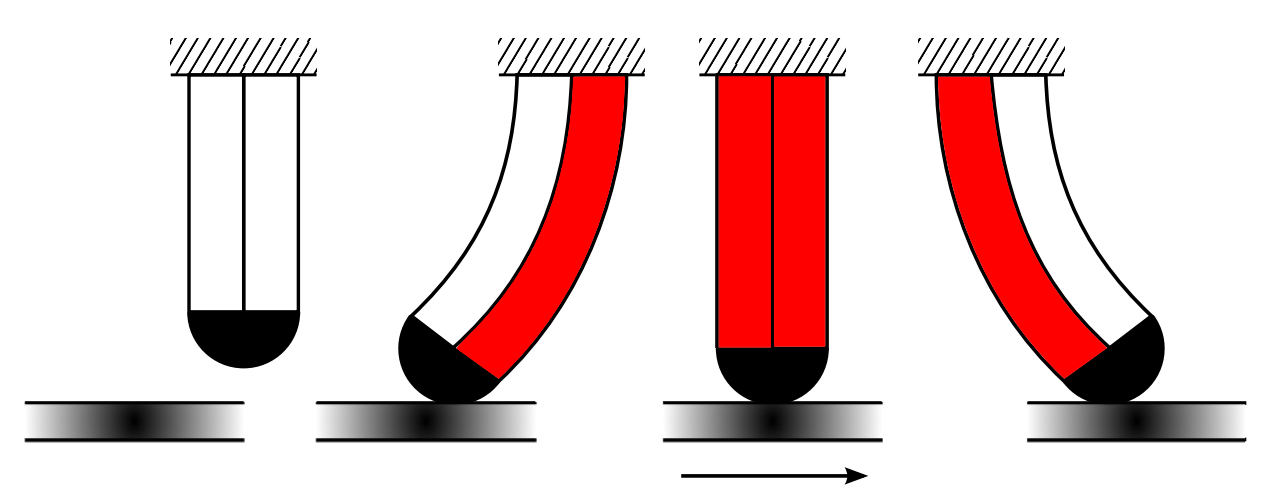

Figure 1. Working principle of a linear motor based on a bi-morph actuator. Step 1: Actuator is unloaded. Step 2: The right material elongates and grips the rod. Step 3: The left material elongates increasingly while the right material retracts which makes the rod move. Step 4: The left material is now elongated alone.

In Andreasen \& Sigmund (2011) the microstructure of saturated poroelastic materials was optimized with respect to the pressure coupling. The obtained material designs made an actuator deflect when pressurized but no direct aims were taken towards maximizing the macroscopic deflection. However, to directly maximize the macroscopic deflection a multiscale approach should be applied as the deflection does not depend on the pressure coupling alone. The stiffness of the structure and the material layup will have a large influence on the performance of the poroelastic actuator as well. This paper therefore presents the theory and application for multiscale modeling of poroelastic actuators along with the optimization of periodic poroelastic materials.

A simple sketch of the problem is shown in figure 2. The actuator consists of two aligned and individually sealed slabs of porous materials which are assembled with a stiff half cylinder of elastic material at the right face and fixed at the left. Alternating the pressure applied to the individual slabs makes it possible to control the actuator in the way indicated in figure 2. The stiff half cylinder is included in order to facilitate a good interaction between actuator and axle and in order to hinder the optimizer in taking advantage of local effects near the end of the actuator. Even a simple bimorph actuator composed of two slabs with isotropic porous materials will perform the task specified above. However, the performance can be significantly improved by altering the material microstructure.

Multi-scale modeling is a common technique within the field of computational mechanics. By assuming that a representative volume element (RVE) can be defined, small material scale details can be included in the computation which would otherwise be impossible to resolve.

Multi-scale methods can be applied to both elastic and inelastic problems. This work takes basis in the asymptotic expansion of the fluid-structure interaction problem (Auriault \& Sanchez-Palencia 1977, Sanchez-Palencia 1980, Auriault et al. 2009). Aspects of the numerical implementation of the homogenization for porous elastic and internally pressurized composites were described in Guedes \& Kikuchi (1990). 


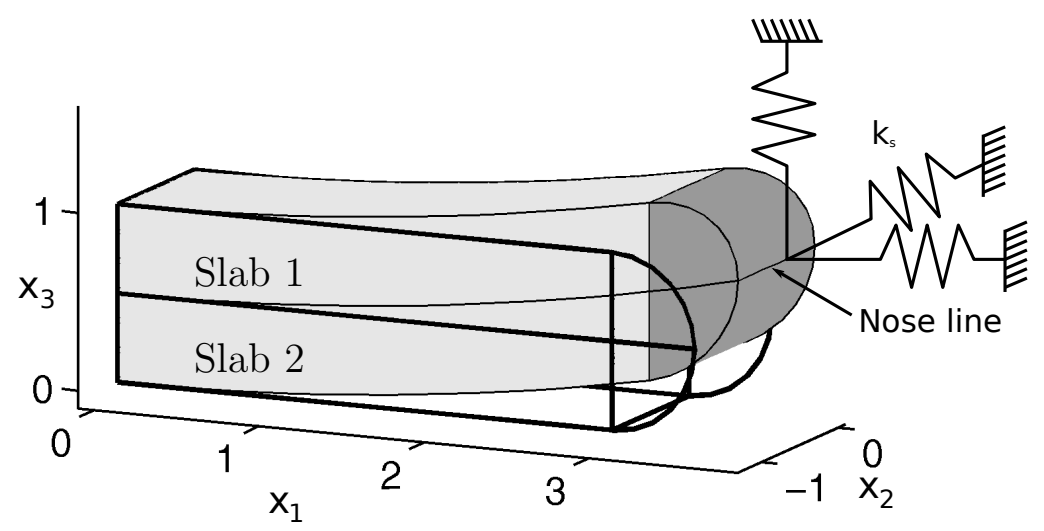

Figure 2. Actuator problem; two individually sealed porous slabs (light gray) assembled with a half cylinder of a stiffer elastic material (dark). Initial and deformed geometry shown. Line springs are attached to the nose line of the actuator.

Terada et al. (1998) give insight in both the asymptotic expansion of the solidfluid mixture problem as well as the implementation. Both a biphasic macroscopic model (poroelasticity, Biot type (Biot 1941)), as used in this work, and a monophasic viscoelastic model is treated therein.

Multi-scale topology optimization is an evolving area in which not only a material distribution problem is optimized but also the material microstructure itself. In fact, this idea was the underlying concept of the seminal paper on topology optimization (Bendsøe \& Kikuchi 1988) where the homogenization approach to topology optimization is presented. By varying the size of the members in a fixed topology microstructure, interpolation functions were obtained for use in the macroscopic distribution problem. This is in contrast to a majority of later applications of topology optimization which rely on the density method, where an interpolation scheme (Bendsøe 1989) is imposed to avoid microstructural considerations during optimization. The underlying microstructure can however be interpreted physically c.f. Bendsøe \& Sigmund (1999).

Elaborating further on the homogenization approach to topology optimization a natural extension is the hierarchical optimization of heterogeneous porous materials meaning a simultaneous optimization of material distribution and material design. Rodrigues et al. (2002) introduced a framework in which the multiple scale designs can be handled. One macroscale and multiple microscale problems are solved iteratively. The methodology was applied to bone remodeling in Coelho et al. (2008) and Coelho et al. (2009). A slightly different approach using interpolation schemes at both the macro and the micro scale were introduced in Liu et al. (2008) for compliance optimization and Nui et al. (2008) for frequency optimization. A two scale method for optimizing the structural compliance by material design and subjected to a seepage constraint was presented by Xu \& Cheng (2010) and Le et al. (2012) presented a method for optimizing material microstructure for macroscopic energy management.

Free material optimization (FMO) introduced in Bendsøe et al. (1994) is yet another approach to multiscale optimization as it is distribution of material parameters 
rather than densities allowing for anisotropic materials. However the method itself does not consider the material designs or composite layups that are needed top realize the optimized local material properties. This inverse homogenization of the stiffness properties was the scope of Sigmund (1994) and Sigmund (1995) and the approach has been further automatized by Schury et al. (2011).

In fluid mechanics, topology optimization was applied to Stokes flow problems in Borrvall \& Petersson (2003) and extended to Navier-Stokes flow in GersborgHansen et al. (2005) and Olesen et al. (2006) using a Brinkman term which introduce an infinitely stiff solid material. The application of topology optimization to fluid structure interaction problems has only recently been approached. Yoon (2010) presented a method for converting the interface stresses to a volume integral which is suited for a monolithic formulation. Kreissl et al. (2010) presented a one way coupling used for the design of flexible micro-fluidic devices. Within optimization of saturated elastic materials the interstitial fluid flow has been considered by imposing conductivity constraints (Sigmund et al. 1998) and by optimizing for the weighted sum of bulk modulus and permeability (Guest \& Prévost 2006). This kind of constraints and considerations applies e.g. for the design of scaffolds for tissue engineering (Hollister 2005).

The fluid-structure interaction is in the present method handled by the homogenization of the Stokes flow in the microstructure which results in a pressure coupling at the macro scale. Contrary to the optimization procedure in Rodrigues et al. (2002) in which both material distribution and design is optimized the multiscale approach taken in this paper deals alone with the material optimization in two predefined subdomains using a macroscopic objective function.

The paper is organized as follows: First an introduction to the model and optimization method is given in section 2. The theory on the two scale expansion of the fluid-structure interaction problem is explained in section 3 presenting the state equations. In section 4 the optimization problem is introduced along with the interpolation functions needed for applying topology optimization to the microstructure design. Section 5 presents the numerical implementation and the results are presented in section 6 . Finally the findings are concluded in section 7 .

\section{Mechanical model and optimization problem}

The approach taken in this paper towards the optimization of a bi-morph pressure driven actuator restricts itself to the design of two porous materials used in the two slabs as illustrated in figure 2 .

To simplify the optimization and modeling procedure we consider only two static load cases which by superposition can approximate the actuator motion. This is possible as we assume the internal flow resistance is low such that the time scale for flow is much smaller than for the actuator motion. This assumption restricts the unit-cell size since very small cells in general have a low permeability and hence a large time scale. Thus 


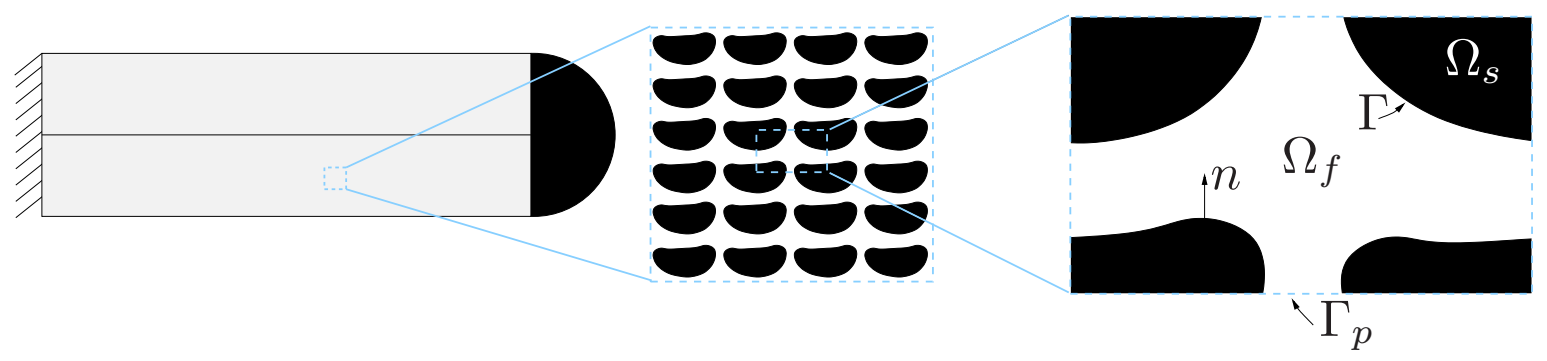

Figure 3. Schematic showing a 2D schematic of the multiscale problem. The macro problem, the actuator, shown left. The periodic porous material in the center and the unit-cell (RVE) to the right. Solid and fluid domains indicated by black and white, interfacing boundaries by $\Gamma$ and periodic boundaries by $\Gamma_{p} . \mathbf{n}$ denotes the unit normal vector to the interface

the actuator concept is less applicable for materials with very small cells.

With the assumption above we can neglect inertia and convection in the fluid phase. Furthermore the pressure will be prescribed in the domains mimicking a steady state solution. Hence, all description of fluid motion can be neglected and only the formulation regarding the solid deformation and pressure induced loading needs to be considered. The theory relies on open-walled structures but no specific measures are taken in order to ensure open cells as all our optimized designs turn out to be openwalled. However, if needed open-walled cells can be ensured by adding permeability constraints as implemented in Andreasen \& Sigmund (2011).

The objective for the optimization study is to maximize the macroscopic deflection of the actuator subject to the two applied pressure load cases. To include the trade-off between stroke and force we apply a set of springs at the end of the actuator which is also shown in figure 2 . The spring stiffness usually influence the stiffness of the obtained design (Sigmund 1997).

The actuator is optimized by altering the microstructure of the two porous material structures by applying topology optimization. In order to do so, the microstructure equations are cast in a monolithic form such that a density is associated to each finite element in the microstructure model representing either solid or fluid. However, as discrete optimization problems are difficult to solve, the problem is relaxed by the introduction of the standard continuous density interpolation approach and solved by a gradient based optimization method.

\section{Theory}

The physical setting is the solid-fluid interaction at the microscale of a saturated periodic porous elastic material. A simplified illustration is shown in figure 3 and the interaction problem can be described as follows.

In the solid elastic material the stress is given by the Cauchy stress tensor 
$\sigma_{s}=\mathbf{E}: \varepsilon(\mathbf{u})$ where $\varepsilon(\mathbf{u})$ is the infinitesimal strain tensor, $\mathbf{u}$ is the displacement vector and $\mathbf{E}$ is the elastic stiffness tensor. The fluid is assumed to be Newtonian and incompressible and the stress is given by $\boldsymbol{\sigma}_{f}=2 \mu D(\mathbf{v})-p \mathbf{I}$ where $D(\mathbf{v})=v_{i j}-\delta_{i j} v_{k k} / 3$ is the strain rate tensor, $p$ the pressure, $\mathbf{I}$ the 2 nd order identity tensor and $\mathbf{v}$ the velocity. Omitting inertial effects, convection and body forces yield the following conservation equations

$$
\begin{array}{ll}
\nabla \cdot \boldsymbol{\sigma}_{s}=\mathbf{0} & \text { in } \Omega_{s} \\
\nabla \cdot \boldsymbol{\sigma}_{f}=\mathbf{0} & \text { in } \Omega_{f} \\
\nabla \cdot \mathbf{v}=0 & \text { in } \Omega_{f}
\end{array}
$$

The boundary conditions between the solid and the fluid requires continuity in the stress over the boundary and the fluid obeys a no-slip condition along the interfacing boundaries. Furthermore, periodicity of displacements, velocities and pressure is required, yielding

$$
\begin{array}{ll}
\left(\boldsymbol{\sigma}_{s}-\boldsymbol{\sigma}_{f}\right) \cdot \mathbf{n}=\mathbf{0} & \text { at } \Gamma \\
\mathbf{v}-\dot{\mathbf{u}}=\mathbf{0} \quad \text { at } \Gamma \\
\mathbf{u}, \mathbf{v}, p \quad \text { periodic } & \text { at } \Gamma_{p}
\end{array}
$$

where $\mathbf{n}$ is the normal vector to the boundary $\Gamma$.

By applying a two scale asymptotic expansion and assuming that the scales are separable this results in a mixed stiffness formulation cf. Wang (2000) which is derived thoroughly in e.g. Auriault et al. (2009). Finally this leaves us with two sets of equations that can be solved in sequence, one describing the microscopic behavior and another describing the macroscopic. As mentioned previously only the steady state solution with a predefined pressure distribution (uniform) will be considered and therefore the macro equation yields

Find $\mathbf{u} \in \mathcal{V}^{3}$ for all $\widehat{\mathbf{u}} \in \mathcal{V}_{0}^{3}$ such that

$$
\int_{V} \varepsilon_{i j}(\widehat{\mathbf{u}}) E_{i j l m}^{H} \varepsilon_{l m}(\mathbf{u}) \mathrm{d} V-\int_{L} \widehat{u}_{i} u_{i} k_{s} \mathrm{~d} L=\int_{V} \alpha_{i j} \varepsilon_{i j}(\widehat{\mathbf{u}}) p \mathrm{~d} V
$$

where $\mathbf{u}$ is displacement, $p$ is pressure and $k_{s}$ is the spring stiffness of the attached springs. These are attached to the nose line $L$ (line perpendicular to 1-3 plane) cf.

figure 2. $E_{i j l m}^{H}$ and $\alpha_{i j}$ are the homogenized stiffness and pressure coupling tensors obtained from the micro model analysis, respectively. The pressure term is located at the right hand side as the pressure distribution is assumed to be known (spatially constant) a priori in the simulations.

\subsection{Micromodel}

The homogenized coefficients that are needed in the poroelastic model are computed by use of homogenization. The deformations of a representative unit cell from the 
periodic microstructure can be obtained using seven different load cases. Afterwards the homogenized coefficients can be computed based on volume averages over the obtained deformations. There are six load cases (three normal and three shear modes) associated with the homogenization of the stiffness tensor $E_{i j l m}^{H}$ and one load case (internal pressure) for the homogenization of the pressure coupling tensor $\alpha_{i j}$.

However, if the basis material is a homogeneous microscopically isotropic elastic material the pressure coupling can be computed directly based on the homogenized stiffness tensor by following e.g. Mei \& Vernescu (2010) and the seventh load case can be neglected.

The analyzed unit cell has periodic boundary conditions and the homogenized stiffness tensor can be computed by solving the following problem

Find $\boldsymbol{\xi}^{k h} \in \mathcal{V}^{3}$ for all $\widehat{\mathbf{u}} \in \mathcal{V}_{0}^{3}$ such that

$$
\begin{aligned}
& \int_{\Omega} \varepsilon_{i j}(\widehat{\mathbf{u}}) E_{i j l m}(\rho) \varepsilon_{l m}\left(\boldsymbol{\xi}^{k h}\right) \mathrm{d} V=\int_{\Omega} E_{i j l m}(\rho) \varepsilon_{l m}^{0, k h} \varepsilon_{i j}(\widehat{\mathbf{u}}) \mathrm{d} V \\
& E_{i j k h}^{H}=\frac{1}{|\Omega|} \int_{\Omega}\left(\varepsilon_{l m}^{0, i j}-\varepsilon_{l m}\left(\boldsymbol{\xi}^{i j}\right)\right) E_{l m p q}(\rho)\left(\varepsilon_{p q}^{0, k h}-\varepsilon_{p q}\left(\boldsymbol{\xi}^{k h}\right)\right) \mathrm{d} \Omega
\end{aligned}
$$

where $\varepsilon^{0, k h}$ is a second order tensor with only a single entry $\varepsilon_{k h}^{0, k h}=1$. The stiffness tensor depends on the design denoted by $\rho$ which will be discussed later. In equation (9) the mutual energies are used to compute the homogenized stiffness tensor.

The pressure coupling tensor can as described also be obtained by homogenization cf. Auriault et al. (2009), however here we utilize the explicit relation between the pressure coupling and homogenized stiffness tensor as described in Mei \& Vernescu (2010) which yields

$$
\alpha_{i j}=\delta_{i j}-\frac{E_{p q i j}^{H} \delta_{p g}}{3 \lambda+2 G}
$$

where $\lambda=E \nu /[(1+\nu)(1-2 \nu)]$ and $G=E /[2(1+\nu)]$ are the Lamé coefficients. For macroscopically isotropic materials the fraction reduces to the ratio between the bulk modulus of the composite and the basis material. This means that the pressure coupling approaches unity if an incompressible basis material is used.

\subsection{Finite element method (FEM)}

The partial differential equations can be solved using the finite element method and for the macroscale equation (7) this yields the following equation system

$$
\mathbf{K u}=\mathbf{Q p}
$$

where $\mathbf{K}$ is the stiffness matrix and $\mathbf{Q}$ is the pressure coupling matrix defined as

$$
\mathbf{K}=\int_{V} \mathbf{B}^{T} \mathbf{E}^{H} \mathbf{B} \mathrm{d} V+\int_{L} k_{s} \mathbf{N}^{T} \mathbf{N} \mathrm{d} L, \quad \mathbf{Q}=\int_{V} \mathbf{B}^{T} \boldsymbol{\alpha} \mathbf{N}_{p} \mathrm{~d} V
$$


where $\mathbf{N}$ and $\mathbf{N}_{p}$ are the shape functions for displacements and pressure, respectively. $\mathbf{B}$ is the strain displacement matrix, $\mathbf{E}^{H}$ is the matrix form of the homogenized stiffness tensor and $\boldsymbol{\alpha}$ is the vector form of the pressure coupling tensor. The stiffness matrix contains contributions from both the elements and the spring stiffnesses. The displacements can easily be found by solving this equation system as the pressure field is assumed to be uniform and given a priori.

For the micro scale problems the discretization yields the equation systems

$$
\breve{\mathbf{K}} \boldsymbol{\xi}^{i}=\breve{\mathbf{f}}^{i}
$$

for $i=1,6$ where $\boldsymbol{\xi}$ is periodic. The stiffness matrix and load vectors are given by

$$
\breve{\mathbf{K}}=\int_{\Omega} \mathbf{B}^{T} \mathbf{E}(\rho) \mathbf{B} \mathrm{d} \Omega, \quad \breve{\mathbf{f}}^{i}=\int_{\Omega} \mathbf{B}^{T} \mathbf{E}(\rho) \mathbf{b}^{i} \mathrm{~d} \Omega
$$

where $\mathbf{E}(\rho)$ is the spatially varying stiffness matrix of the base material and $\mathbf{b}^{i}$ corresponds to the i'th column of the $6 \times 6$ identity matrix. This formulation is the FE-discretization of (8).

\section{Optimization}

The goal for the optimization of the poroelastic actuator is to maximize its vertical deflection such that the performance of a poroelastic linear motor can be improved. This is done by imposing topology optimization in the material design process in which the strict distinction between solid and void is relaxed by the introduction of a design interpolation function. At design convergence the densities are supposed to be either 0 or 1 due to the imposed penalization of intermediate densities.

Considering the actuator displayed in figure 2 having a movement as illustrated in figure 4 the performance is quantified by the average vertical deflection $\bar{u}_{3}$ which is the vertical deflection integrated along the nose line. Assuming a linear response to the pressurization of either domain, the optimization is based on the two solutions corresponding to pressurization of each domain. These two load cases are referred to by a superscript parenthesized number.

In order to apply topology optimization to the unit cell design, each element in the FE-discretization is given a density $\rho_{e} \in[0 ; 1]$ such that it can represent values between pure fluid $\left(\rho_{e}=0\right)$ and solid $\left(\rho_{e}=1\right)$. During the optimization intermediate densities are allowed and the stiffness is interpolated using the RAMP scheme (Stolpe \& Svanberg 2001)

$$
\zeta\left(\rho_{e}\right)=\left(10^{-4}+\frac{\rho_{e}\left(1-10^{-4}\right)}{1+p\left(1-\rho_{e}\right)}\right)
$$

where $p$ is a penalization factor, here $p=3$. For the stiffness this yields $\mathbf{E}(\rho)=\zeta(\rho) \mathbf{E}_{0}$ where $\mathbf{E}_{0}$ is the stiffness tensor of the isotropic basis material. This scheme imposes a lower bound on the stiffness which avoids the equation system being singular and 

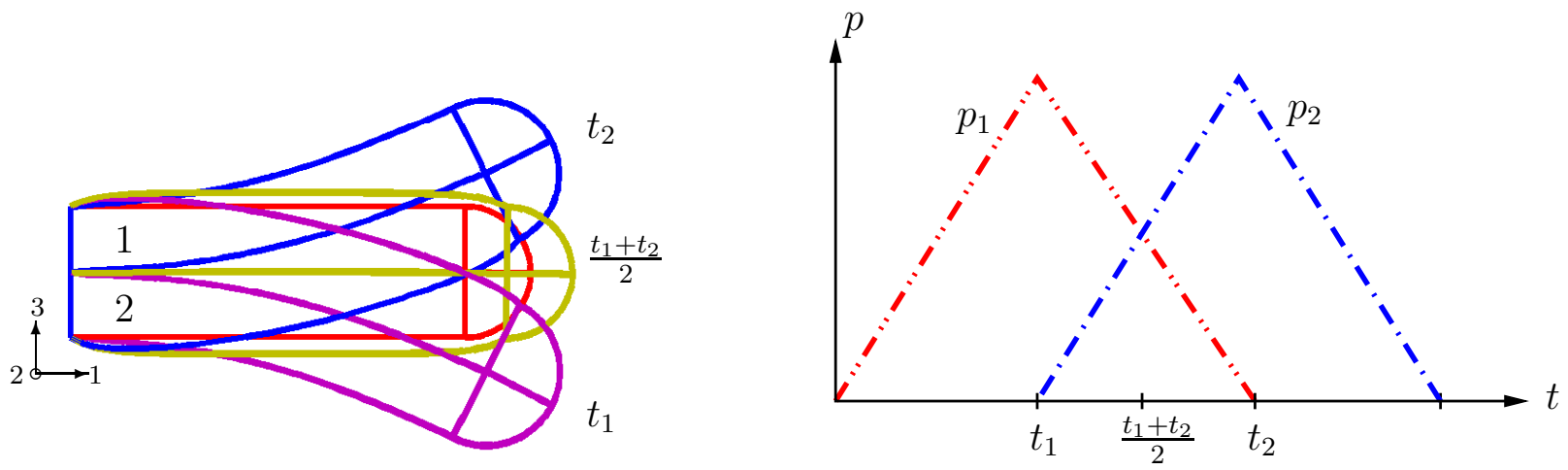

Figure 4. Left: Sketch of actuator motion path. Right: Pressure-time relation for the domains. First the upper domain(1) is loaded by a pressure, which then decays linearly while the lower domain(2) is loaded by a linearly increasing pressure. Finally this pressure is also decreased and the actuator returns to its initial state.

furthermore ensures a non-zero gradient for $\rho_{e}=0$. This design dependence of the stiffness applies to the micro problem (8) and the volume averaging (9).

The optimization problem can be formulated as a min-max optimization problem:

$$
\left.\begin{array}{rl}
\min _{\boldsymbol{\rho} \in \mathbb{R}^{N}} & \max \left\{\bar{u}_{3}^{(1)},-\bar{u}_{3}^{(2)}\right\} \\
\text { s.t. } & \text { Macro equation } \\
& \text { Micro equations } \\
& \sum_{e=1}^{N_{1}} v_{e} \rho_{e}-\gamma_{1} V_{0} \leq 0, \\
& \sum_{e=N_{1}+1}^{N_{2}} v_{e} \rho_{e}-\gamma_{2} V_{0} \leq 0, \\
& g_{i} \leq 0 \quad \text { for } e=1, \ldots, N
\end{array}\right\}
$$

where $\bar{u}_{3}$ denotes the average deflection in the 3rd direction i.e. the vertical deflection integrated along the nose line. $N=N_{1}+N_{2}$ is the number of elements (total and in each material), $\gamma$ is the allowed solid volume fraction for each material, $v_{e}$ is the element volume, $V_{0}$ is the total volume of the unit-cell and $g_{i}=g_{i}(\boldsymbol{\rho}, \mathbf{u})$ denotes a set of inequality constraints which will be introduced later.

\subsection{Sensitivity analysis}

The stiffness matrix $\mathbf{K}$ of the macro problem depends on the homogenized stiffness tensor that again depends on the design variables. This also holds for the pressure coupling matrix $\mathbf{Q}$ that depends on the homogenized pressure coupling tensor. Therefore the sensitivity of the objective function must be computed by the chain rule as

$$
\frac{\mathrm{d} \Phi}{\mathrm{d} \rho_{e}}=\frac{\partial \Phi}{\partial \mathbf{E}^{H}} \frac{\mathrm{d} \mathbf{E}^{H}}{\mathrm{~d} \rho_{e}}+\frac{\partial \Phi}{\partial \boldsymbol{\alpha}} \frac{\mathrm{d} \boldsymbol{\alpha}}{\mathrm{d} \rho_{e}}, \quad \text { for } e=1, \ldots, N
$$


In order to compute the first factor of both terms an adjoint sensitivity analysis (Michaleris et al. 1994) of the macroscopic equations can be performed. First, the Lagrangian $\mathcal{L}$ is defined by the objective function, the adjoint variable and the residual as

$$
\mathcal{L}=\Phi+\boldsymbol{\lambda}^{T}(-\mathbf{K u}+\mathbf{Q p})
$$

The sensitivities with respect to the stiffness are computed by differentiating the Lagrangian with the stiffness matrix

$$
\begin{aligned}
\frac{\mathrm{d} \mathcal{L}}{\mathrm{d} \mathbf{E}^{H}} & =\underbrace{\frac{\partial \Phi}{\partial \mathbf{E}^{H}}+\boldsymbol{\lambda}^{T} \frac{\partial(-\mathbf{K u}+\mathbf{Q p})}{\partial \mathbf{E}^{H}}}_{X} \\
& +\underbrace{\left(\frac{\partial \Phi}{\partial \mathbf{u}}+\boldsymbol{\lambda}^{T} \frac{\partial(-\mathbf{K u}+\mathbf{Q p})}{\partial \mathbf{u}}\right)} \frac{\mathrm{d} \mathbf{u}}{\mathrm{d} \mathbf{E}^{H}}
\end{aligned}
$$

To eliminate the $X$-marked terms we choose $\boldsymbol{\lambda}$ as the solution to the adjoint problem

$$
\mathbf{K}^{T} \boldsymbol{\lambda}=\frac{\partial \Phi}{\partial \mathbf{u}}
$$

Having solved this problem for $\boldsymbol{\lambda}$, the sensitivities are obtained from the remaining terms of $(15)$

$$
\frac{\mathrm{d} \mathcal{L}}{\mathrm{d} \mathbf{E}^{H}}=\frac{\partial \Phi}{\partial \mathbf{E}^{H}}-\boldsymbol{\lambda}^{T} \frac{\partial \mathbf{K}}{\partial \mathbf{E}^{H}} \mathbf{u}
$$

Analogously, the sensitivity with respect to the pressure coupling tensor can be obtained as

$$
\frac{\mathrm{d} \mathcal{L}}{\mathrm{d} \boldsymbol{\alpha}}=\frac{\partial \Phi}{\partial \boldsymbol{\alpha}}+\boldsymbol{\lambda}^{T} \frac{\partial \mathbf{Q}}{\partial \boldsymbol{\alpha}} \mathbf{p}
$$

using the same adjoint solution as above.

\subsection{Micro model sensitivities}

The derivation of the micromodel sensitivities, i.e. the sensitivity of the components in the homogenized stiffness tensor, is described in Sigmund \& Torquato (1997) and they yield

$$
\frac{\mathrm{d} E_{i j k h}^{H}}{\mathrm{~d} \rho_{e}}=\frac{1}{|\Omega|} \int_{\Omega_{e}}\left(\varepsilon_{l m}^{0, i j}-\varepsilon_{l m}\left(\boldsymbol{\xi}^{i j}\right)\right) E_{l m p q}^{\prime}\left(\rho_{e}\right)\left(\varepsilon_{p q}^{0, k h}-\varepsilon_{p q}\left(\boldsymbol{\xi}^{k h}\right)\right) \mathrm{d} \Omega
$$

where ()$^{\prime}$ denotes the partial derivative with respect to the design variable. The sensitivities of $\boldsymbol{\alpha}$ can be computed, by differentiating (10), as

$$
\frac{\mathrm{d} \alpha_{k h}}{\mathrm{~d} \rho_{e}}=-\frac{\mathrm{d} E_{i j k h}^{H}}{\mathrm{~d} \rho_{e}} \frac{\delta_{i j}}{3 \lambda+2 G}
$$



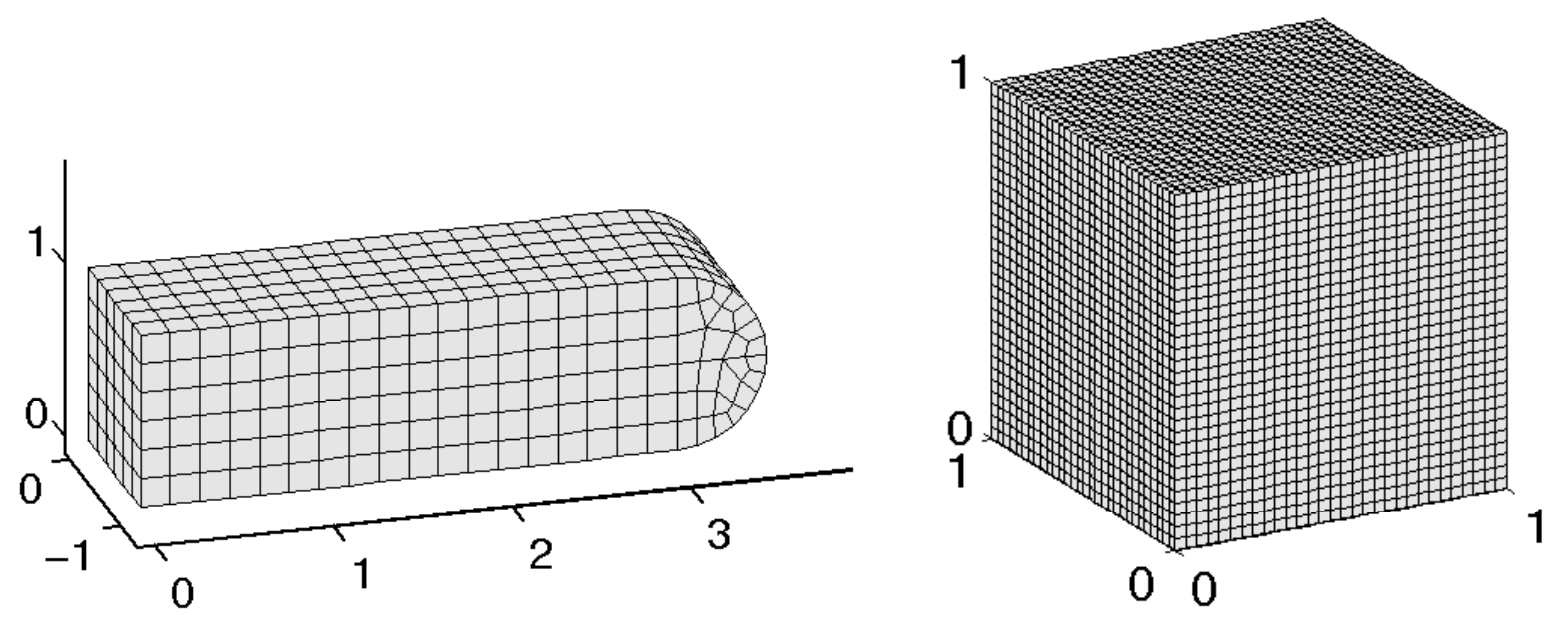

Figure 5. The meshes used in the analysis: a) Macro mesh, 810 hexahedral elements. b) Micro mesh with $30^{3}$ brick elements.

\section{Numerical implementation}

An optimization software has been written in the framework of Comsol Multiphysics 3.5a and Matlab 2008b. The macroscopic modeling of the actuator is done using the FEM functionality within Comsol using 2nd order hexahedral elements. The mesh used for the macroscopic model is shown in figure 5(a). The solution is obtained using the built-in geometric multi grid (GMG) preconditioned conjugate gradient (PCG) method.

The homogenization procedure has been written solely in Matlab using 1st order regular hexahedral elements in order to get the highest possible resolution. The mesh is shown in figure 5(b). All faces have periodic boundary conditions and they are enforced using the same node numbers on the opposing sides of the unit-cell. In order to prevent rigid body motion a single node has been fully constrained. The solution is obtained using an incomplete Choleskey PCG method.

The optimization problem is solved using the method of moving asymptotes (MMA) by Svanberg (1987) and the convergence criteria used is $\left\|\boldsymbol{\rho}^{i}-\boldsymbol{\rho}^{i-1}\right\|_{\infty}<0.001$. The nonsmooth min-max problem formulation is converted into a smooth problem by using a bound formulation.

Furthermore a standard density filter (Bruns \& Tortorelli 2001, Bourdin 2001) has been used in order to avoid checkerboard patterns. To try to avoid convergence to local minima we use a continuation strategy for the filter radius $R$ such that its final value is 1.4 times the element side length and the filter uses a linear weighting.

The optimization procedure can be summarized as follows 
(i) Initialize

(ii) While $\left\|\boldsymbol{\rho}^{i}-\boldsymbol{\rho}^{i-1}\right\|_{\infty}<0.001$ and $R_{\min } \leq R$

(a) Solve micro problems, compute $\mathbf{E}^{H}, \boldsymbol{\alpha}, \frac{\mathrm{d} \mathbf{E}^{H}}{\mathrm{~d} \boldsymbol{\rho}}, \frac{\mathrm{d} \boldsymbol{\alpha}}{\mathrm{d} \boldsymbol{\rho}}$,

Eq. (8), (9),(10),(19),(20)

(b) Solve macro problem, compute $\Phi$, Eq. (7)

(c) Solve adjoint macro problem, compute $\frac{\partial \Phi}{\partial \mathbf{E}^{H}}, \frac{\partial \Phi}{\partial \alpha}$,

Eq. (16),(17),(18)

(d) Find sensitivities using chain rule, $\frac{\mathrm{d} \Phi}{\mathrm{d} \boldsymbol{\rho}}=\frac{\partial \Phi}{\partial \mathbf{E}^{H}} \frac{\mathrm{d} \mathbf{E}^{H}}{\mathrm{~d} \boldsymbol{\rho}}+\frac{\partial \Phi}{\partial \boldsymbol{\alpha}} \frac{\mathrm{d} \boldsymbol{\alpha}}{\mathrm{d} \boldsymbol{\rho}}$, Eq. (13)

(e) Update $\boldsymbol{\rho}$ using MMA

(f) Decrease $R$ if $\left\|\boldsymbol{\rho}^{i}-\boldsymbol{\rho}^{i-1}\right\|_{\infty}<0.001$

(iii) Post process

\section{Results}

The numerical studies comprise four test cases: 1) a comparison with the method from Andreasen \& Sigmund (2011) for a single material actuator; 2) a two material design; 3) a two material design with constraints on the extension/deflection ratio, and 4) a torsional actuator. All optimization problems are subject to a $30 \%$ volume constraint $(\gamma=0.3)$. The volume constraints turns out to be active in all examples except if stated otherwise. The elastic basis material has the properties $E=1$ and $\nu=0.3$.

\subsection{One material design}

In the paper by Andreasen \& Sigmund (2011) a method for indirect optimization of a single material actuator was presented. This was done under the assumption that the lateral deflection could be maximized by increasing the magnitude of the corresponding entry in the pressure coupling tensor, e.g. increasing deflection in the 3rd direction by maximizing the magnitude of $\alpha_{13}$. Using the same code and formulation as in the reference, a material is generated for which $\alpha_{13}$ is minimized (i.e. maximized in downward direction). The result is shown in figure 6(a).

The material unit cell plotted in figure 6(b) is obtained using the macro problem as it is presented in figure 2 with springs attached to the nose line having the stiffness $k_{s}=10^{-3}$. The optimized material design does not completely resemble that of the minimized $\alpha_{13}$ entry. The fact that the material is also extending due to the internal pressure makes it impossible to rely on the shear effects alone. The inclined planes are still present but a very thick layer of material ensures stiffness in the 1-direction such that the pressure is not wasted on deforming the extension spring, when it is the vertical deflection that matters.

By detaching the springs from the right edge the design changes and this is shown in figure $6(\mathrm{c})$. Not having any springs means that no force must be transmitted and thereby no or only small stiffness is required. It is clearly seen from the material design that not all material is utilized which of course also is evident when evaluating the 


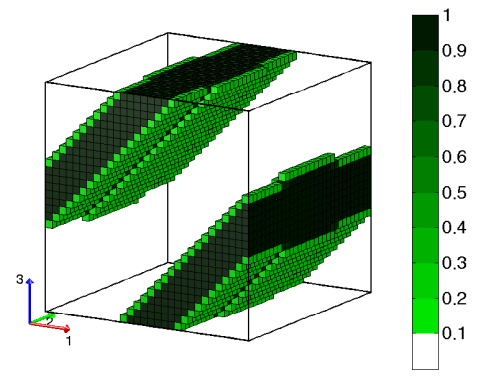

(a)

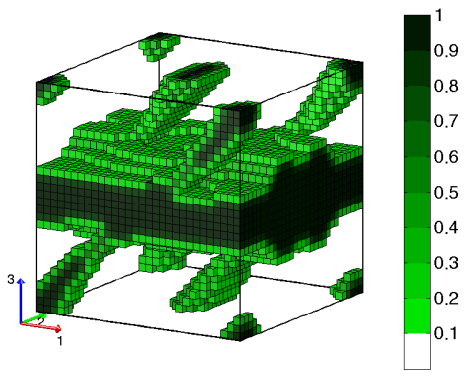

(b)

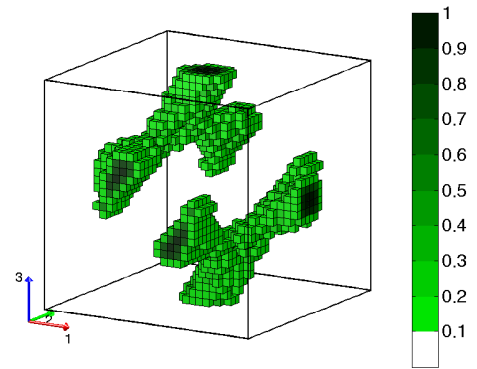

(c)

Figure 6. (a) Material with minimized $\alpha_{13}$ component. (b) Resulting material from maximizing the vertical displacement of a single material actuator with springs with stiffness $k_{s}=10^{-3}$ attached. (c) As (b) but with no springs attached.

\begin{tabular}{lrrr}
\hline Figure & $\alpha_{13}$ & $\bar{u}_{3},\left(k_{s}=10^{-3}\right)$ & $\bar{u}_{3},\left(k_{s}=0\right)$ \\
\hline $6(\mathrm{a})$ & $-\mathbf{0 . 0 6 0 2}$ & -10.36 & -77.56 \\
$6(\mathrm{~b})$ & -0.0027 & $\mathbf{6 4 6 . 2 4}$ & -6858.45 \\
$6(\mathrm{c})$ & -0.0002 & -10.42 & $-\mathbf{1 6 1 6 6 . 6 5}$ \\
\hline
\end{tabular}

Table 1. Cross check for optimized single material actuators shown in figure 6 . The post evaluation was subjected to a pressure of $p=1$. The minimum value for each column is emphasized.

stiffness and pressure coupling. A comparison of the performance of the three designs is listed in table 1. It is seen that they individually perform best for the problem formulation for which they were optimized. The rather poor macroscopic performance of the pressure-coupling-optimized material having parallel planes was discussed in Andreasen \& Sigmund (2011) and for the final designs stiffness constraints were added in order to ensure stable and load transmitting materials.

\subsection{Two material design}

This section considers the design of a bi-morph poroelastic actuator. The objective is to make the actuator move as illustrated in figure 4, namely extension and downwards deflection followed by an upwards deflection. The figure also presents a pressure-time relation which indicates the sequence in which the domains are pressurized. First the upper domain is pressurized. This is followed by a linear pressure decrease in the upper domain and an increase in lower domain until the lower domain is fully pressurized. Removing the pressure the actuator moves back to its initial configuration.

As described in section 4 the optimization will only consider two quasi static load cases. One for the pressurization of each domain. Figure 7 shows the designs obtained using the formulation in equation (12). The initial design was a solid cross and the final result has been obtained using a continuation approach on the filter radius $(R=\{2$, 1.4 \} elements side length). From $7(\mathrm{a})$ and $7(\mathrm{c})$ it is seen that the material unit cell 
has a main beam in the 1st direction (along the actuator) while the connections in the 2nd and 3rd direction are rather thin. This results in a low transversal stiffness of the material. The resulting stiffness and pressure coupling matrices of the two materials are:

$$
\begin{aligned}
& \mathbf{E}_{1}^{H}=\left[\begin{array}{rrrrrr}
25.527 & 0.153 & 0.175 & -0.000 & 0.262 & 0.000 \\
& 0.128 & 0.102 & 0.000 & 0.150 & -0.000 \\
& & 0.148 & -0.000 & 0.182 & 0.000 \\
& & & 0.055 & 0.000 & 0.061 \\
\text { sym } & & & & 0.296 & 0.000 \\
& & & & & 0.097
\end{array}\right] \cdot 10^{-2} \\
& \boldsymbol{\alpha}_{1}=\left[\begin{array}{rrr}
0.897 & -0.000 & -0.002 \\
& 0.998 & -0.000 \\
\text { sym } & & 0.998
\end{array}\right] \\
& \mathbf{E}_{2}^{H}=\left[\begin{array}{rrrrrr}
25.527 & 0.153 & 0.175 & -0.000 & -0.262 & -0.000 \\
& 0.128 & 0.102 & -0.000 & -0.150 & -0.000 \\
& & 0.148 & 0.000 & -0.182 & -0.000 \\
& & & 0.055 & 0.000 & -0.061 \\
\text { sym } & & & & 0.296 & 0.000 \\
& & & & & 0.097
\end{array}\right] \cdot 10^{-2}, \\
& \boldsymbol{\alpha}_{2}=\left[\begin{array}{rrr}
0.897 & -0.000 & 0.002 \\
& 0.998 & -0.000 \\
\text { sym } & & 0.998
\end{array}\right]
\end{aligned}
$$

from which it is seen that the material properties are symmetric around the $\left(x_{1}, x_{2}\right)$ plane. No explicit symmetry constraint is enforced but starting with a symmetric initial design and optimizing using a symmetric objective function does not leave any motivation to the optimizer to make two different designs.

Figure 7(b) and 7(d) illustrate the deformed actuator. The in- and out-of-plane deformations are small as they do not directly enter the objective. It is seen that the deflection is dominating the deformation. The upper and lower boundaries are nearly straight which indicates that the shear strain is important. This is linked to the low transversal stiffness of the materials together with the pressure coupling which allows the actuator to deflect. In order to explore the nature of the deformation the stress state can be analyzed. The total stress $\boldsymbol{\sigma}=\mathbf{E}^{H} \boldsymbol{\varepsilon}(\mathbf{u})-\boldsymbol{\alpha} p$ in point $\mathbf{x}_{\text {low }}=(1.5,-0.5,0.25)$ and $\mathbf{x}_{u p}=(1.5,-0.5,0.75)$ while pressurizing the lower domain is

$\boldsymbol{\sigma}_{\mathbf{x}_{\text {low }}}=\left\{\begin{array}{r}-1.548 \\ 1.001 \\ 0.987 \\ -0.001 \\ -2.283 \\ 0.001\end{array}\right\} \cdot 10^{-3}-\left\{\begin{array}{r}0.897 \\ 0.998 \\ 0.998 \\ -0.000 \\ 0.002 \\ -0.000\end{array}\right\} \cdot 10^{-3}=\left\{\begin{array}{r}-2.444 \\ 0.003 \\ -0.011 \\ -0.001 \\ -2.286 \\ 0.001\end{array}\right\} \cdot 10^{-3}, \quad \sigma_{\mathbf{x}_{u p}}=\left\{\begin{array}{r}2.714 \\ 0.097 \\ 0.016 \\ -0.000 \\ 0.455 \\ -0.000\end{array}\right\} \cdot 10^{-3}$

and it is seen that $\sigma_{1}$ and $\sigma_{5}$ in the lower domain have the same magnitude. As seen in figure 8 the actuator only moves vertically while pressurized. 


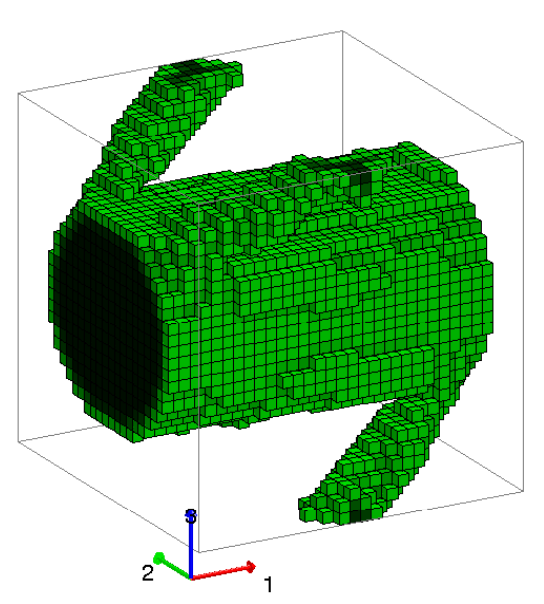

(a) Material 1

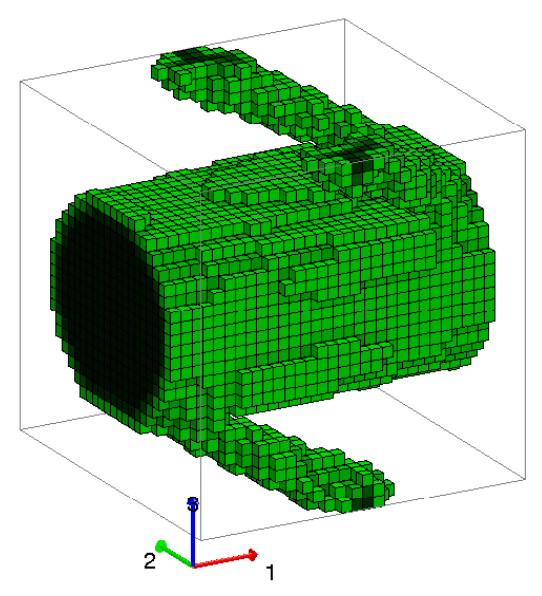

(c) Material 2
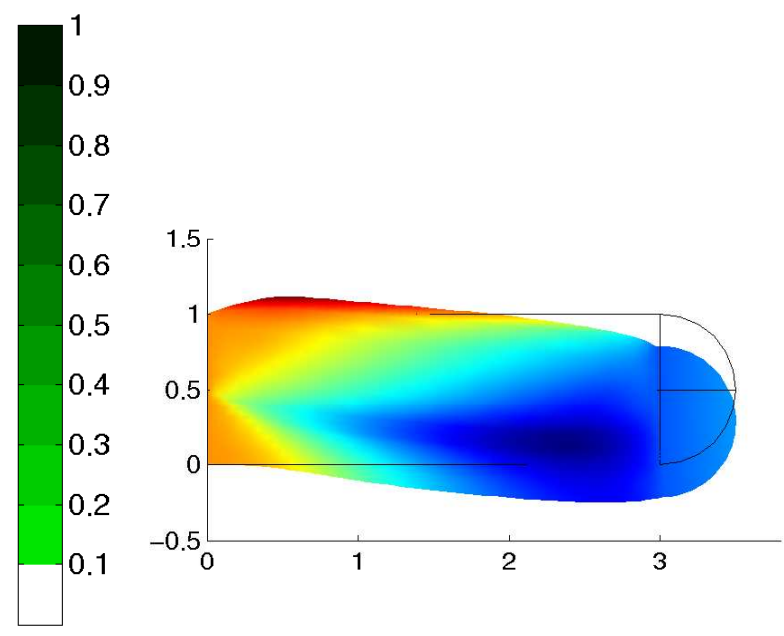

(b) Deformation load case 1. Color: $u_{3}$
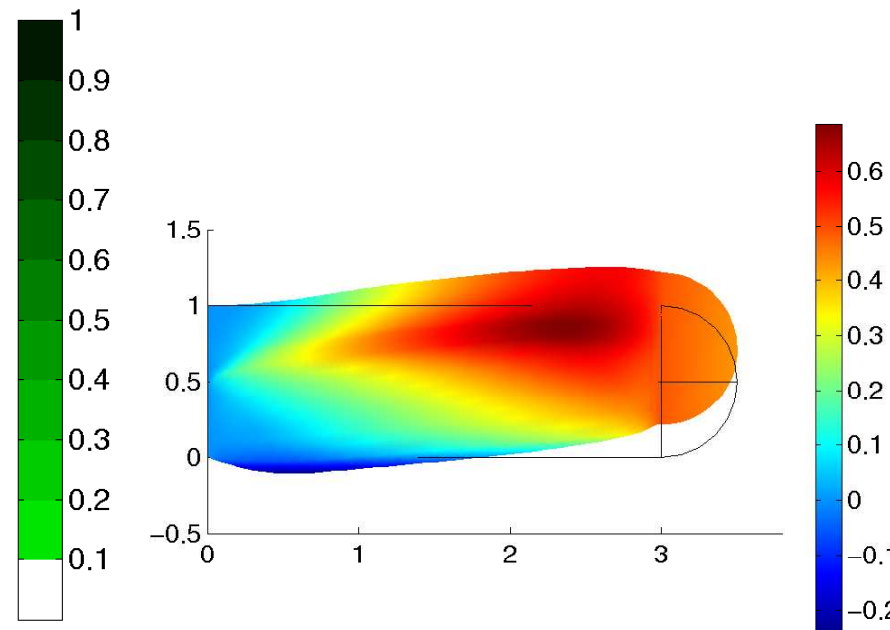

(d) Deformation load case 2. Color: $u_{3}$

Figure 7. Optimized material configuration and deflections for each of the two load cases. Optimized with spring stiffness $k=10^{-3}$. Same deformation scaling apply to both plots. $\Phi_{\text {final }}=\max \left\{-\bar{u}_{3}^{(1)}, \bar{u}_{3}^{(2)}\right\}=0.424, p=10^{-3}$
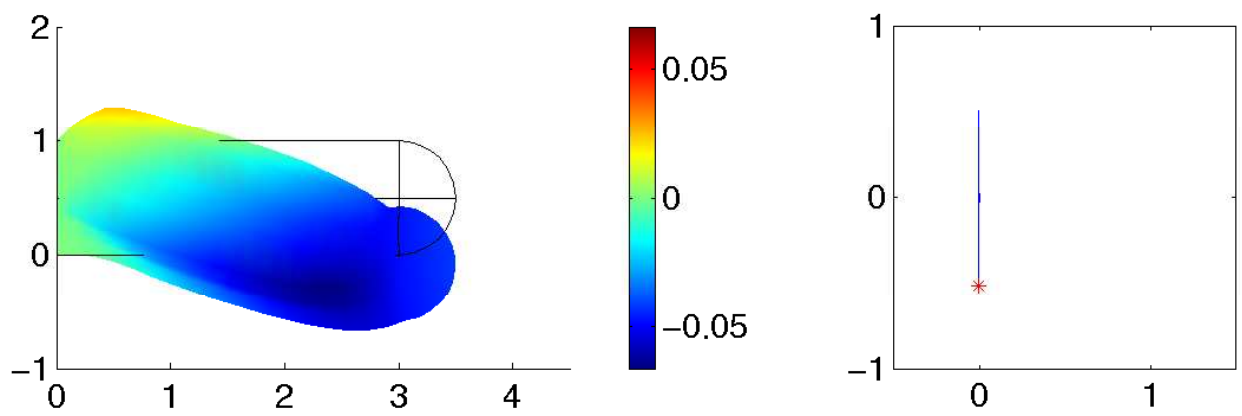

Figure 8. Left: Deformed actuator with pressurized upper domain. Right: Motion path with indication of current state. Deformations are exaggerated. 
6.2.1. Localization Having computed the macroscopic response the corresponding deformation of the unit cell can be investigated. In general (Auriault et al. 2009) the micro deformation is given by

$$
u_{i}^{(1)}=\xi_{i}^{l m} \varepsilon_{l m}\left(\mathbf{u}^{(0)}\right)-\eta_{i} p^{(0)}+\bar{u}_{i}^{(1)}(\mathbf{x})
$$

where the first two terms are linear combinations of the homogenization equation solutions and the macroscopic strain and pressure, respectively. The final term is a rigid body movement of the unit-cell. However as the macro and micro structures have a finite scale ratio a uniform straining of the unit cell also needs to be superposed to the local deformations. This uniform straining corresponds to the macroscopic strain field applied to the unit cell

$$
u_{i}^{\operatorname{def}}(\mathbf{y}, \mathbf{x})=\varepsilon_{i j}\left(\mathbf{u}^{(0)}\right) y_{j}
$$

where $\mathbf{y}$ the non-dimensional local coordinate. This deformation field is superposed on that of equation (26). The unit cell deformations corresponding to the design and loads shown in figure 7 are shown in figure 9 .
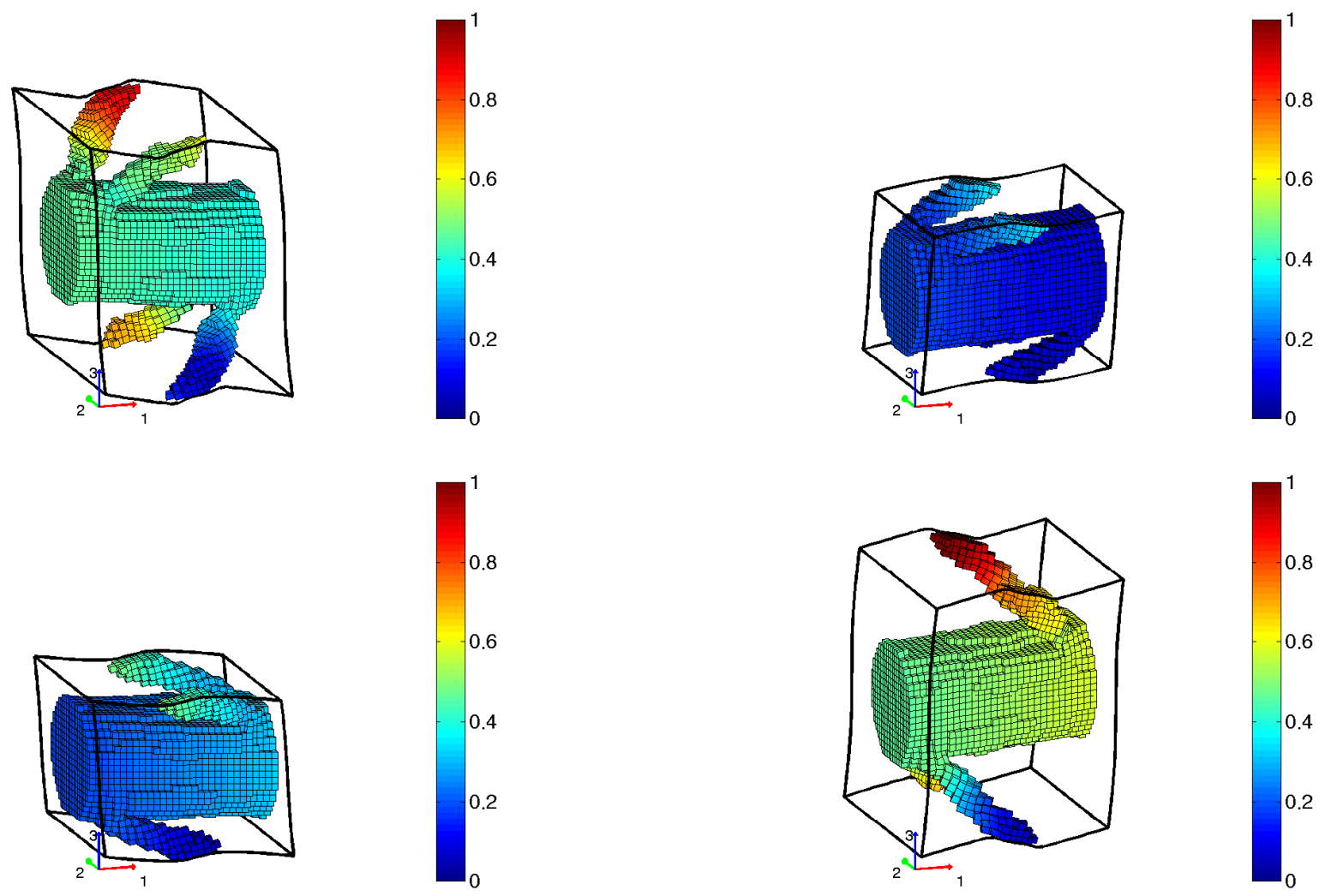

Figure 9. Deformed unit cells for the two materials (top and bottom) and for each load case (left/right). Color shows the deformation vector sum $\left(\sqrt{u_{1}^{2}+u_{2}^{2}+u_{3}^{2}}\right)$. The unit cell has a side length of 0.01 and the deformations are scaled by a factor 0.5. The unit-cells are located in the same points as used for the stress evaluation; $\mathbf{x}_{\text {low }}=(1.5,-0.5,0.25)$ and $\mathbf{x}_{u p}=(1.5,-0.5,0.75)$ 


\subsection{Deflection/extension constraints}

The previous actuator shown in figure 7 performs very well with respect to vertical deflection for which the design was optimized. However in order to use the actuator in a linear motor an extension is required. This can be ensured by adding a set of constraints that restrict the ratio of the extension and the deflection to be approximately one. The constraints are given by

$$
\begin{aligned}
& g_{1}=\left(-u_{3}^{(1)} / u_{1}^{(1)}-1\right)^{2}-\epsilon \leq 0 \\
& g_{2}=\left(u_{3}^{(2)} / u_{1}^{(2)}-1\right)^{2}-\epsilon \leq 0
\end{aligned}
$$

where $\epsilon$ is a small number (here $\epsilon=0.01$ ).

Taking these constraints into consideration the material design changes and the resulting material structures are shown in figure 10. Again (a) and (c) show the material microstructures and (b) and (d) the deformations. It is clearly seen that the actuator now also extends. In comparison to the deformations of the previous actuator it seems to bend more than it shears. From figure 10(a) and (c) it is seen that the solid connection along the 1st axis is it getting very thin, approximately $1 / 15$ of

the cell side length, which decrease the directional stiffness drastically. Also, figure 12 indicates that the centerpart of the microstructure rotates in a mechanism-like fashion, in turn resulting in the controlled macroscopic deflection pattern. Both these effects are reflected in the reduced stiffness of this microstructure compared to the one without deflection/extension constraints.

The material parameters yield:

$$
\begin{aligned}
& \mathbf{E}_{1}^{H}=\left[\begin{array}{rrrrrr}
2.811 & -0.186 & -0.266 & 0.000 & -0.280 & 0.000 \\
& 0.113 & 0.049 & -0.000 & 0.041 & -0.000 \\
& & 0.088 & -0.000 & 0.056 & -0.000 \\
& & & 0.071 & -0.000 & 0.051 \\
& & & & 0.064 & -0.000 \\
\text { sym } & & & & & 0.063
\end{array}\right] \cdot 10^{-2} \\
& \boldsymbol{\alpha}_{1}=\left[\begin{array}{rrr}
0.991 & -0.000 & 0.001 \\
& 1.000 & -0.000 \\
\text { sym } & & 1.001
\end{array}\right] \\
& \mathbf{E}_{2}^{H}=\left[\begin{array}{rrrrrr}
2.811 & -0.186 & -0.266 & -0.000 & 0.280 & 0.000 \\
& 0.113 & 0.049 & 0.000 & -0.041 & -0.000 \\
& & 0.088 & 0.000 & -0.056 & -0.000 \\
& & & 0.071 & -0.000 & -0.051 \\
\text { sym } & & & & 0.064 & 0.000 \\
& & & & & 0.063
\end{array}\right] \cdot 10^{-2} \\
& \boldsymbol{\alpha}_{2}=\left[\begin{array}{rrr}
0.991 & -0.000 & -0.001 \\
& 1.000 & 0.000 \\
\text { sym } & & 1.001
\end{array}\right]
\end{aligned}
$$




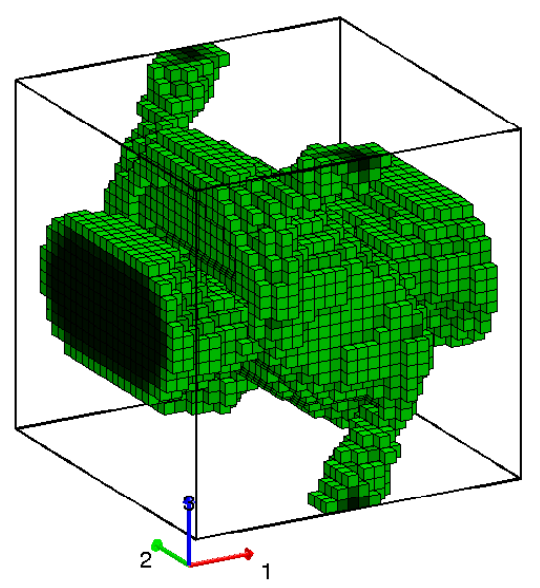

(a) Material 1

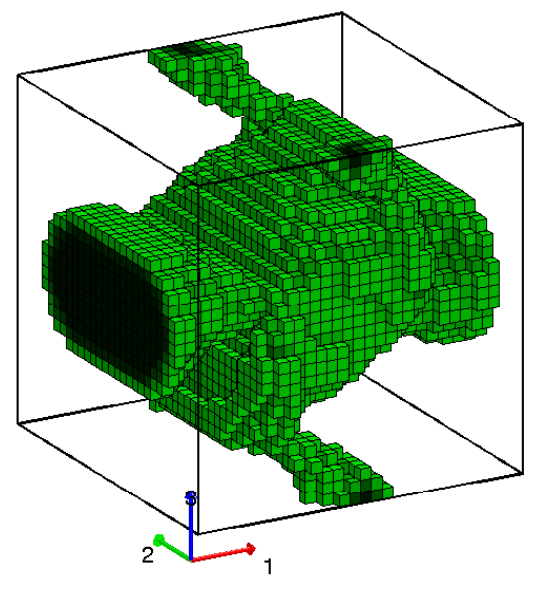

(c) Material 2

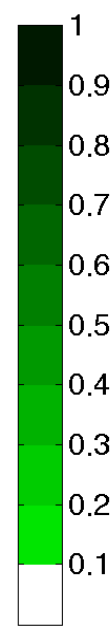

(b) Deformation load case 1. Color: $u_{3}$
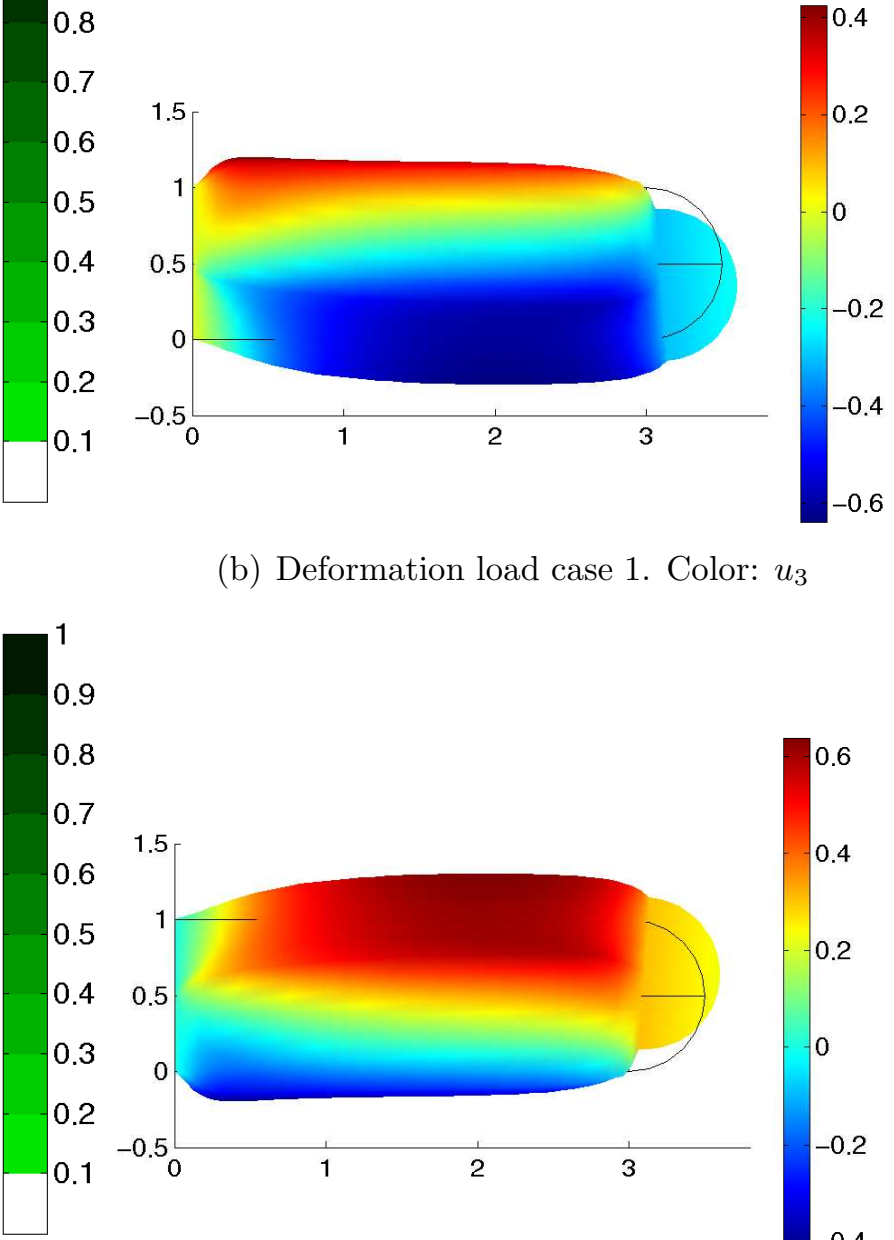

(d) Deformation load case 2. Color: $u_{3}$

Figure 10. Optimized material configuration and deflections for each of the two load cases. A set of constraints ensure that the extension-deflection ratio is approximately one. Optimized with spring stiffness $k=10^{-3}$. Same deformation scaling apply to both plots. $\Phi_{\text {final }}=\max \left\{-\bar{u}_{3}^{(1)}, \bar{u}_{3}^{(2)}\right\}=0.226, p=10^{-3}$

This is also revealed if the stress state is investigated:

$\boldsymbol{\sigma}_{\mathbf{x}_{\text {low }}}=\left\{\begin{array}{r}-1.534 \\ 0.978 \\ 0.969 \\ 0.001 \\ -0.920 \\ -0.000\end{array}\right\} \cdot 10^{-3}-\left\{\begin{array}{r}0.991 \\ 1.000 \\ 1.001 \\ 0.000 \\ -0.001 \\ -0.000\end{array}\right\} \cdot 10^{-3}=\left\{\begin{array}{r}-2.524 \\ -0.022 \\ -0.032 \\ 0.001 \\ -0.919 \\ -0.000\end{array}\right\} \cdot 10^{-3}, \quad \sigma_{\mathbf{x}_{u p}}=\left\{\begin{array}{r}1.468 \\ 0.071 \\ 0.018 \\ 0.001 \\ -0.102 \\ 0.001\end{array}\right\} \cdot 10^{-3}$

If the pressure is varied linearly as shown in figure 4 the actuator deflects as shown in figure 11. The actuator is shown in a state where the upper domain is pressurized 

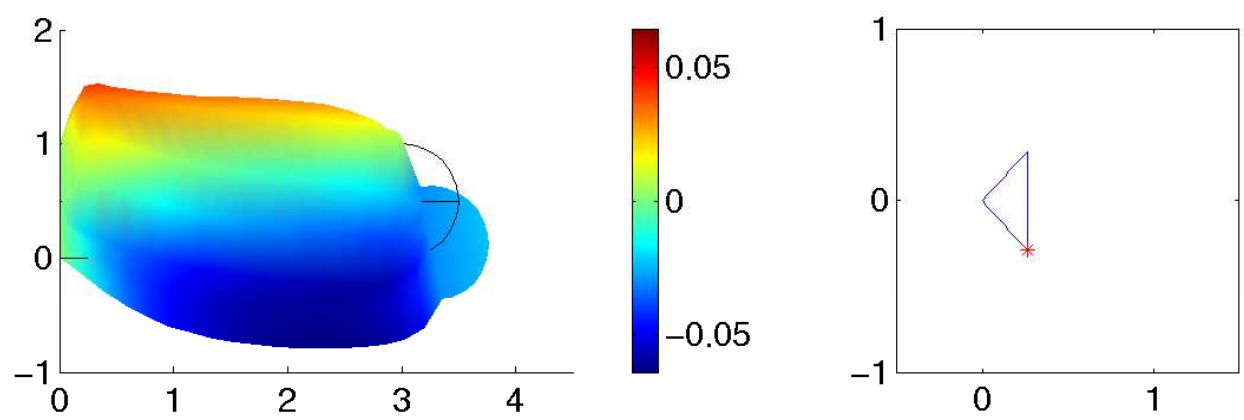

Figure 11. Left: Deformed actuator with pressurized upper domain. Right: Motion path with indication of current state. Deformations are exaggerated.
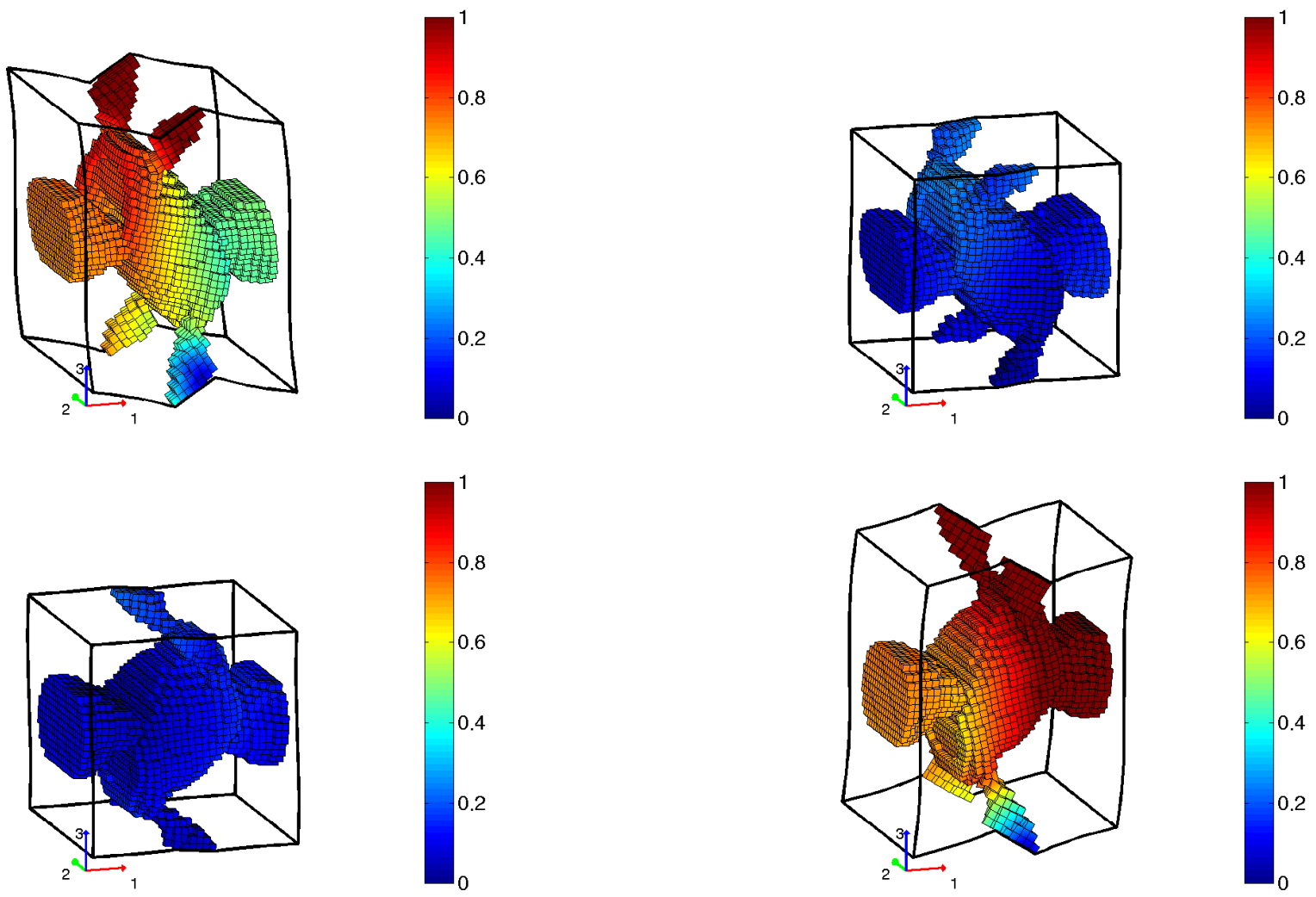

Figure 12. Deformed unit cells for the two materials (top and bottom) and for each load case (left/right). Color shows the deformation vector sum $\sqrt{u_{1}^{2}+u_{2}^{2}+u_{3}^{2}}$. Deformations are scaled by a factor 0.25 . The unit-cells are located in the same points as the stresses are evaluated; $\mathbf{x}_{\text {low }}=(1.5,-0.5,0.25)$ and $\mathbf{x}_{u p}=(1.5,-0.5,0.75)$

and the computed motion path is shown in the right figure. 

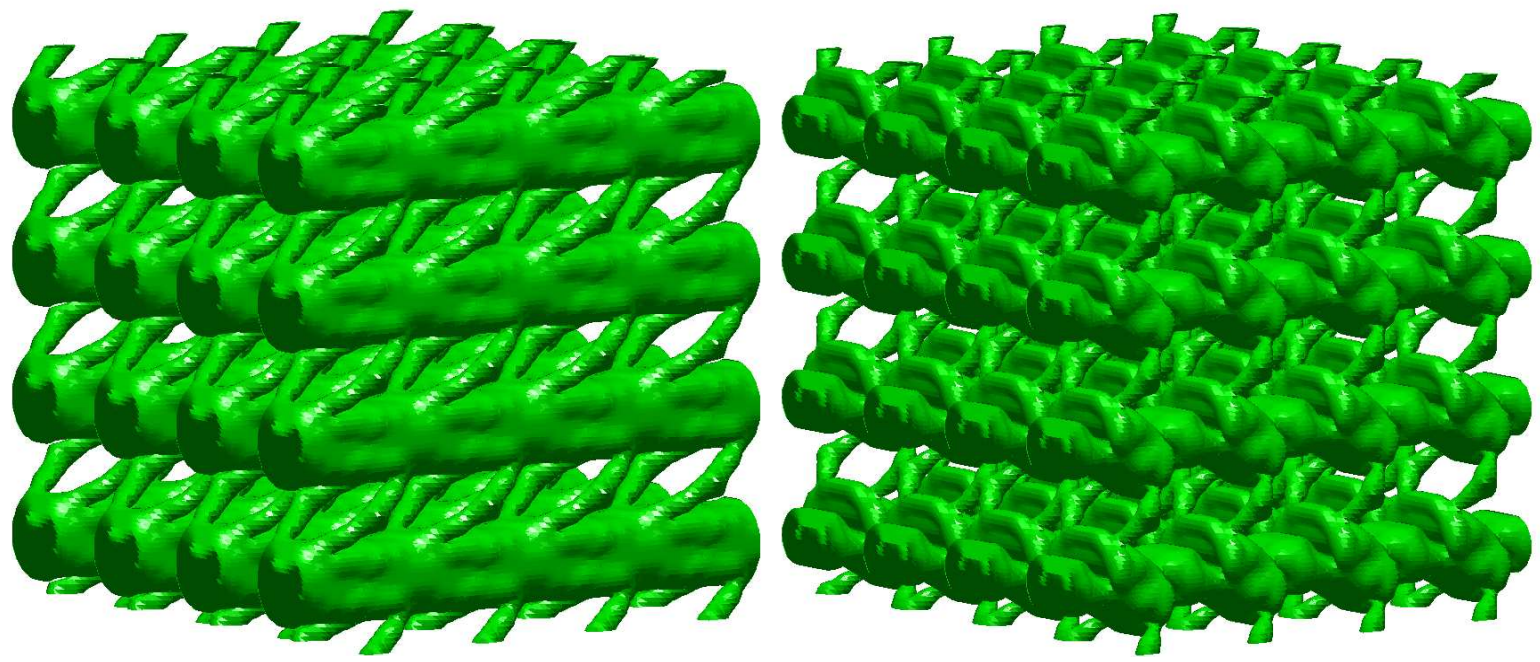

Figure 13. Smoothed and repeated unit cells for the optimized material 1 shown in figure 7 and 10
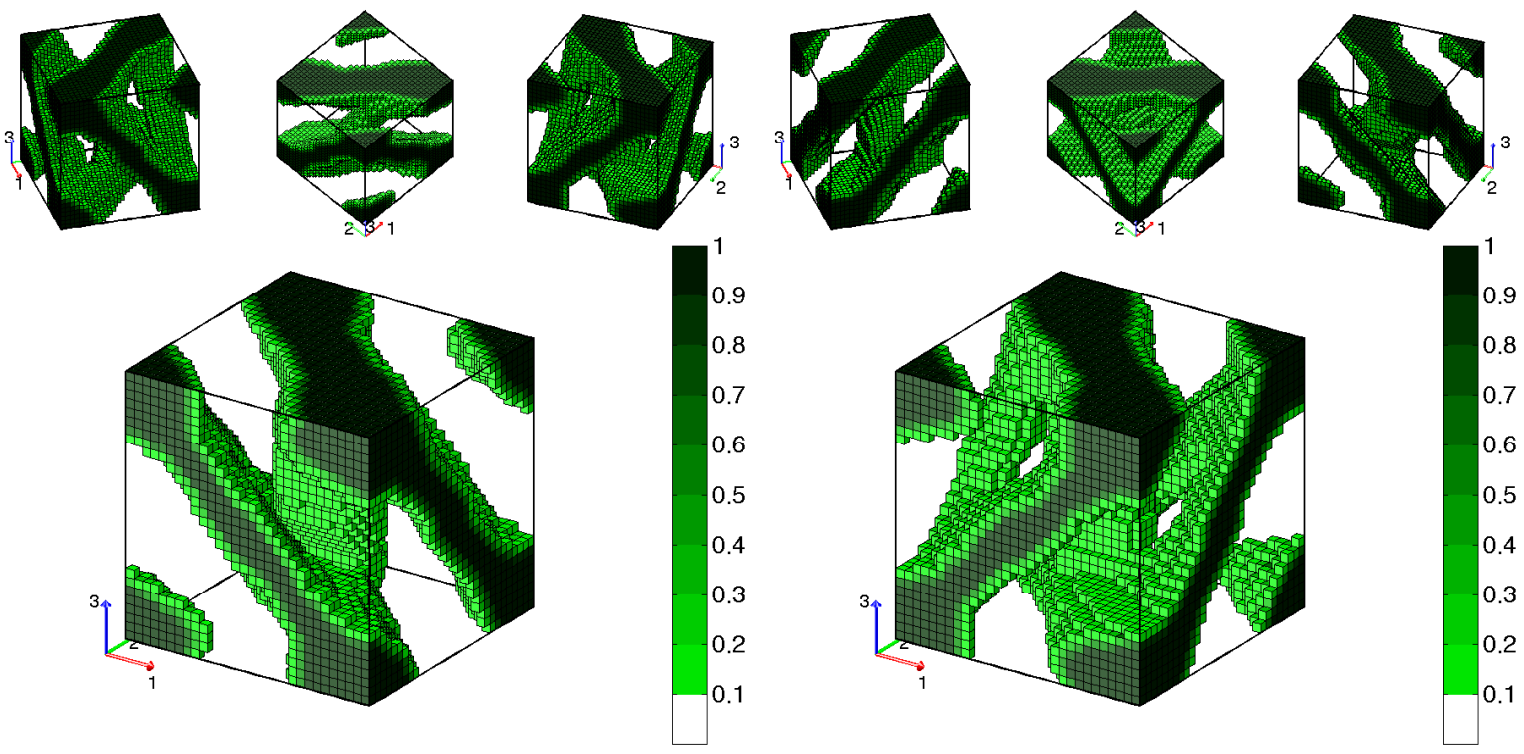

(a) Material 1

(b) Material 2

Figure 14. Optimized materials for the torsional actuator. Optimized using spring stiffness $k=10^{3}$, and $p=1 \Phi_{\text {final }}=-2.527 \cdot 10^{-4}$

\subsection{Torsion actuator}

By changing the objective function slightly a torsion actuator can be generated i.e. one that rotates around its longitudinal axis. The modified objective function is

$$
\Phi=\max \left\{\int_{L} u_{3}^{(1)}\left(x_{2}+0.5\right) \mathrm{d} L, \quad-\int_{L} u_{3}^{(2)}\left(x_{2}+0.5\right) \mathrm{d} L\right\}
$$




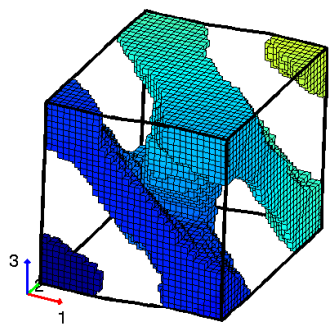

(a) Upper
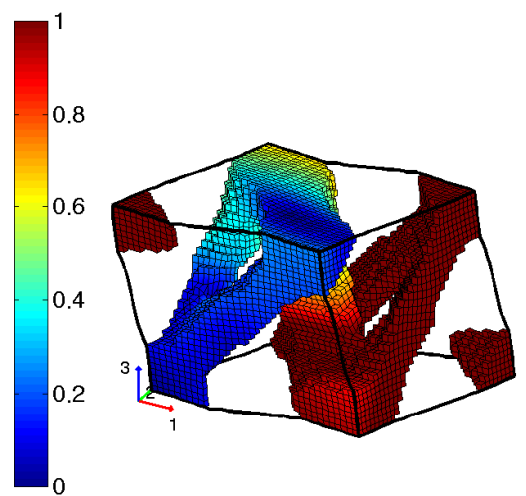

(b) Lower

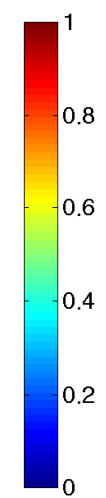

Figure 15. Deformed unit cells for the two materials for load case 2 only. Color shows the deformation vector sum $\sqrt{u_{1}^{2}+u_{2}^{2}+u_{3}^{2}}$. Deformations are scaled by a factor 0.25 . The unit-cells are located in the same points as previously; $\mathbf{x}_{l o w}=(1.5,-0.5,0.25)$ and $\mathbf{x}_{u p}=(1.5,-0.5,0.75)$
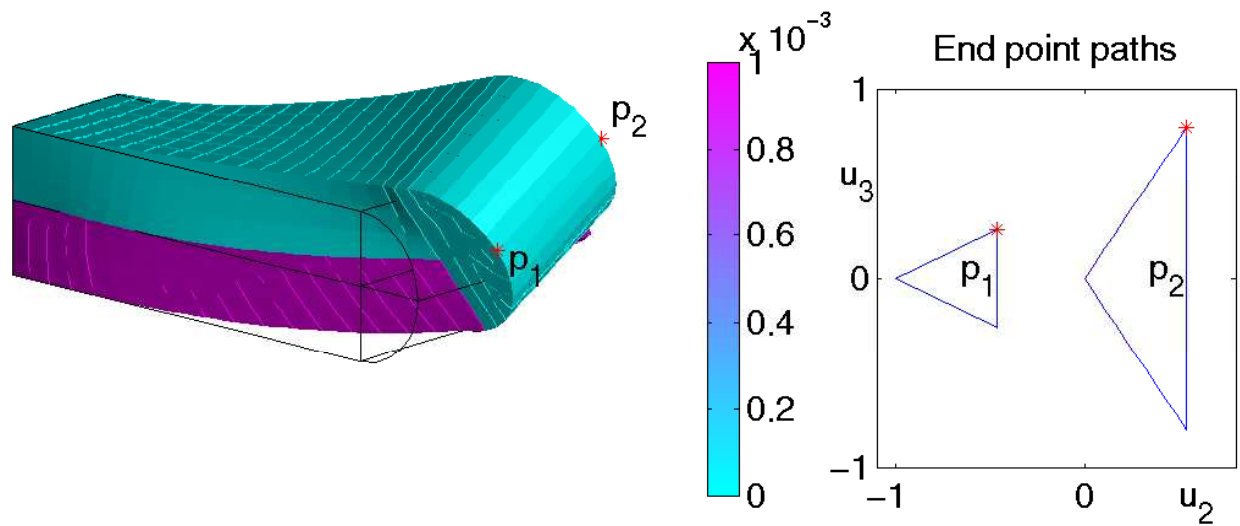

Figure 16. Left: Deformed actuator with pressurized lower domain. Right: Motion path of nose line nodes with indication of current state in $\left(x_{2}, x_{3}\right)$-plane. Deformations are exaggerated.

where $x_{2}$ is the coordinate along the nose-line. In this way one side of the actuator preferably moves upwards while the other moves downwards creating a rotational motion. The optimized microstructures are shown in figure 14 and it is seen that they contain inclined planes with an interconnection and a hole. Similar structures were obtained and treated in Andreasen \& Sigmund (2011) but contrary to this case they were constrained to impose a minimum stiffness and permeability. The objective for the two problems differ, however the mechanism that leads to the rotation seems to be the same, namely an extension of the microstructure along the diagonal which is normal to the inclined planes. This is seen from the deformed microstructures of load case 2 shown in figure 15. The microscopic deformation is shown in figure 16 along with the deformation-path $\left(\left(x_{2}, x_{3}\right)\right.$-plane $)$ of the two nodes defining the nose-line. It is evident that the actuator rotates, however for larger rotations another objective function should 
be considered along with geometric non-linearities.

\section{Conclusion}

This paper presents an approach to material design for use in poroelastic actuators based on multiscale modeling and topology optimization.

The optimization of a single material multiscale modeled actuator was compared with the results obtained for optimizing the pressure coupling coefficient of the microstructure alone. A bi-morph poroelastic actuator was optimized subject to a volume constraint and a set of additional constraints was imposed in order to ensure both extension and deflection of the actuator. Furthermore a torsion actuator was optimized which yielded a material with inclined and interconnected planes similar to those obtained in Andreasen \& Sigmund (2011).

A future extension includes the possibility of making hierarchical optimization in which either each macroscopic element or region of elements have individual material configurations in the fashion of Rodrigues et al. (2002). By employing the poroelastic model in a bone remodeling process it would be possible to study the direct impact of internal fluid flow on the design of the scaffolds as opposed to the indirect approach by Coelho et al. (2009). This work may also be extended to dynamic problems using the full Biot equations (Biot 1956).

\section{Acknowledgments}

This research was conducted within the DCAMM Research School through a grant from the Danish Agency for Science, Technology and Innovation. The authors would like

to thank the members of the TopOpt research group (www.topopt.dtu.dk) for fruitful discussions. 


\section{References}

Andreasen C S \& Sigmund O 2011 Structural and Multidisciplinary Optimization 43, 693-706.

Auriault J L, Boutin C \& Geindreau C 2009 Homogenization of Coupled Phenomena in Heterogeneous Media ISTE Ltd and John Wiley \& Sons Inc.

Auriault J L \& Sanchez-Palencia E 1977 Journal De Mecanique 16(4), 575-603.

Bendsøe M P 1989 Structural and Multidisciplinary Optimization 1, 193-202.

Bendsøe M P, Guedes J M, Haber R B, Pedersen P \& Taylor J E 1994 Journal of Applied Mechanics 61(4), 930-937.

Bendsøe M P \& Kikuchi N 1988 Computer Methods in Applied Mechanics and Engineering 71(2), 197224.

Bendsøe M P \& Sigmund O 1999 Archive of Applied Mechanics 69, 635-654.

Biot M A 1941 Journal of Applied Physics 12(2), 155-164.

Biot M A 1956 The Journal of the Acoustical Society of America 28(2), 168-178.

Borrvall T \& Petersson J 2003 International Journal for Numerical Methods in Fluids 41(1), 77-107.

Bourdin B 2001 International Journal for Numerical Methods in Engineering 50, 2143-2158.

Bruns T \& Tortorelli D 2001 Computer Methods in Applied Mechanics and Engineering 190, 3443-3459.

Coelho P G, Fernandes P R, Guedes J M \& Rodrigues H C 2008 Structural and Multidisciplinary Optimization 35(2), 107-115.

Coelho P G, Fernandes P R, Rodrigues H C, Cardoso J B \& Guedes J M 2009 Journal of Biomechanics 42(7), 830-837.

Friend J, Umeshima A, Ishii T, Nakamura K \& Ueha S 2004 Sensors and Actuators A: Physical $\mathbf{1 0 9}(3), 242-251$.

Gersborg-Hansen A, Sigmund O \& Haber R 2005 Structural and Multidisciplinary Optimization 30, 181-192.

Guedes J \& Kikuchi N 1990 Computer Methods in Applied Mechanics and Engineering 83(2), 143-198.

Guest J K \& Prévost J H 2006 International Journal of Solids and Structures 43(22-23), 7028-7047.

Hollister S J 2005 Nat Mater 4(7), 518-524.

Kreissl S, Pingen G, Evgrafov A \& Maute K 2010 Structural and Multidisciplinary Optimization 42, 495-516.

Le C, Bruns T E \& Tortorelli D A 2012 Journal of Mechanics and Physics of Solids 60, 351-378.

Liu L, Yan J \& Cheng G 2008 Computers \& Structures 86(13-14), 1417 - 1425.

Mei C C \& Vernescu B 2010 Homogenization methods for multiscale mechanics World Scientific Singapore; Hackensack, NJ.

Michaleris P, Tortorelli D A \& Vidal C A 1994 Int. J. Numer. Meth. Engng. 37(14), 2471-2499.

Nui B, Jun Y \& Cheng G 2008 Structural and Multidisciplinary Optimization 39(2), 115-132.

Olesen L H, Okkels F \& Bruus H 2006 International Journal for Numerical Methods in Engineering 65(7), 975-1001.

Rodrigues H, Guedes J \& Bendsøe M 2002 Structural and Multidisciplinary Optimization 24, 1-10.

Sanchez-Palencia E 1980 Non-homogeneous media and vibration theory Lecture Notes in Physics, 127. Berlin Heidelberg New York: Springer- Verlag.

Schury F, Stingl M \& Wein F 2011 submitted to SIAM journal of Scientific Computing .

Sigmund O 1994 International Journal of Solids and Structures 31(17), 2313-2329.

Sigmund O 1995 Mechanics of Materials 20(4), 351-368.

Sigmund O 1997 Mechanics of Structures and Machines 25(4), 493-524.

Sigmund O \& Torquato S 1997 Journal of the Mechanics and Physics of Solids 45(6), 1037-1067.

Sigmund O, Torquato S \& Aksay I A 1998 Journal Of Materials Research 13(4), 1038-1048.

Stolpe M \& Svanberg K 2001 Structural and Multidisciplinary Optimization 22(2), 116-124.

Svanberg K 1987 International Journal for Numerical Methods in Engineering 24(2), 359-373.

Terada K, Ito T \& Kikuchi N 1998 Computer Methods in Applied Mechanics and Engineering 153, 223257. 


\section{CONCLUSION}

Wang H F 2000 Theory of Linear Poroelasticity with Applications to Geomechanics and Hydrogeology Princeton University Press.

Xu S \& Cheng G 2010 Structural and Multidisciplinary Optimization 41(4), 575-587.

Yoon G H 2010 International Journal for Numerical Methods in Engineering 82(5), 591-616. 